\title{
CARACTERIZAÇÃO FÍSICA DOS FRUTOS E SEMENTES DA ESPÉCIE Byrsonima gardneriana A. JuSS NO SEMIÁRIDO ALAGOANO
}

\author{
Élida Monique da Costa Santos \\ Universidade Federal de Alagoas, Instituto de Geografia, Desenvolvimento e Meio Ambiente, \\ Maceió, AL, Brasil \\ elidamoniquecs@outlook.com
}

Kallianna Dantas Araujo

Universidade Federal de Alagoas, Instituto de Geografia, Desenvolvimento e Meio Ambiente, Pós-Graduação em Geografia, Maceió, AL, Brasil kallianna.araujo@igdema.ufal.br

Mayara Andrade Souza

Centro Universitário CESMAC, Pós-Graduação em Análise de Sistemas Ambientais, Maceió, AL, Brasil mayarandrade@hotmail.com

\begin{abstract}
Ana Beatriz da Silva
Universidade Federal de Alagoas, Instituto de Geografia, Desenvolvimento e Meio Ambiente, Programa de PósGraduação em Geografia, Maceió, AL, Brasil anabtrizsilva@gmail.com

Elba dos Santos Lira Universidade Federal de Alagoas, Instituto de Geografia, Desenvolvimento e Meio Ambiente, Maceió, AL, Brasil elbaslira@yahoo.com.br

Danúbia Lins Gomes Universidade Federal de Alagoas, Instituto de Ciências Biológicas e da Saúde, Pós-Graduação em Diversidade Biológica e Conservação nos Trópicos, Maceió, AL, Brasil dlinsgomes@yahoo.com.br

João Gomes da Costa Centro Universitário CESMAC, Pós-Graduação em Análise de Sistemas Ambientais, Maceió, AL, Brasil joao-gomes.costa@embrapa.br
\end{abstract}

\begin{abstract}
RESUMO
A espécie Byrsonima gardneriana A. Juss (Murici) possui fruto pequeno, amareloalaranjado, com aroma e sabor marcantes, considerado fonte de alimento rica em nutrientes. Objetivou-se analisar as características físicas dos frutos e sementes da espécie B. gardneriana em duas localidades, em Olho D’Água do Casado, Alagoas. Foram coletados 1.000 frutos maduros em dois locais (Sítio Alto - P1 e Sítio Pudrinhos - P2), selecionados 250 unidades, separando os visualmente sadios, inteiros e sem deformação, tomando-se uma amostra de 150 unidades e mensurados: peso dos frutos e sementes, rendimento de polpa e casca, rendimento das sementes, comprimento e diâmetro dos frutos e das sementes. Os frutos e sementes do Ponto 2 (Sítio Pudrinhos) são mais desenvolvidos em relação ao Ponto 1 (Sítio Alto) para as variáveis diâmetro longitudinal e transversal dos frutos e sementes, peso dos frutos e das sementes e peso de polpa e casca, atribuída a variabilidade edafoclimática existentes entre os locais estudados; $A$ espécie $B$. gardneriana possui semente grande em relação ao tamanho do fruto, não havendo elevado rendimento de polpa. Entretanto, não diminui sua importância para a indústria alimentícia, pois, essa característica pode ser melhorada por intermédio da seleção de plantas com frutos que apresentam maior rendimento de polpa.
\end{abstract}

Palavras-chave: Caatinga. Espécies nativas. Malpighiaceae. Rendimento de polpa e casca. 
Caracterização física dos frutos e sementes da espécie byrsonima gardneriana a. juss no semiárido alagoano
Élida Monique da Costa Santos; Kallianna Dantas Araujo; Mayara Andrade Souza; Ana Beatriz da Silva; Elba dos Santos Lira; Danúbia Lins Gomes; João Gomes da Costa

\title{
PHYSICAL CHARACTERIZATION OF FRUITS AND SEEDS OF Byrsonima gardneriana A. Juss, IN THE ALAGOAN SEMI-ARID
}

\begin{abstract}
The Bysonima gardneriana A. Juss (Murici) species, has yellow-orange small fruit, with remarkable aroma and flavor, considered a nutritionally rich food source. The objective of this study was to analyze the physical characteristics of the fruits and seeds of $B$. gardneriana species located in Olho D'Água do Casado, Alagoas. A total of 100 mature fruits were collected at two sites (Sítio Alto - P1 and Sítio Pudrinhos - P2). Then, 250 units were selected according to the following visual criteria: healthy fruits, unbroken fruit and without deformation fruits, also 150 units were selected to measure weight of fruits and seeds, yield of pulp and peel, seed yield, fruit's and seed's length and diameter. According to the variables longitudinal and transverse diameter of fruits and seeds, fruits and seeds weight, pulp and bark weight, the fruits and seeds from Sítio Pudrinhos were more developed than the ones collected at Sítio Alto due to the edaphoclimatic variability among the studied sites. The species $B$. gardneriana has large seed in comparison to its fruit size, there is no high yield of pulp. However, it does not diminish its importance for the food industry, since this characteristic can be improved by the selection of plants with fruits with higher yield of pulp.
\end{abstract}

Keywords: Caatinga. Native species. Malpighiaceae. Yield of pulp and bark.

\section{INTRODUÇÃO}

Benini et al. (2010), mencionam que as plantas nativas são aquelas que ocorrem naturalmente em determinado ambiente possuindo elevada importância para o ecossistema local. De acordo com Drumond (2000) a flora nativa da Caatinga apresenta espécies com características anatômicas, morfológicas e funcionais especializadas para a sobrevivência em diferentes condições de clima e solo, típicos desta fisionomia vegetal. O Murici (Byrsonima gardneriana A. Juss) pertence ao gênero Byrsonima, com registro de espécies no Norte, na região Central, nas regiões Serranas do Sudeste e encontradas também na região Nordeste (ROLIM, 2009). Seixas et al. (2011) levantaram 35 espécies pertencente ao gênero Byrsonima, distribuídos nos Estados de Alagoas, Bahia, Ceará, Maranhão, Pernambuco, Piauí, Sergipe e Rio Grande do Norte. Das espécies levantadas nesses Estados, 57\% são nativas/endêmicas do Brasil, encontradas principalmente nos domínios fitogeográficos da Mata, Cerrado, Caatinga. Em Alagoas foram encontradas: B. crispa, B. gardneriana, B. sericea, B. stipulaceae e $B$. verbascifolia.

Chitarra e Chitarra (1990) afirmam que as características físicas dos frutos devem ser analisadas em conjunto, já que isoladamente são pouco representativas, sendo fundamental para a definição de técnicas de manuseio pós-colheita, como para a boa aceitação do produto pelo consumidor, pois, a qualidade dos frutos é conferida aos atributos de qualidade, dentre os quais que respondem pela aparência externa, destacam-se sabor, odor, textura, tamanho e forma do fruto que acrescido ao valor nutritivo, são características que apresentam importância para o produtor, para o mercado na forma de produtos processados (industrializados) e o consumidor.

Diante do exposto, elaborou-se o seguinte questionamento: existe diferença entre as características físicas dos frutos e sementes da espécie Byrsonima gardneriana A. Juss (Murici) em duas localidades, em Olho D'Água do Casado, Alagoas? Para responder a esse questionamento foi elaborada a seguinte hipótese: as características físicas dos frutos e sementes da espécie Byrsonima gardneriana A. Juss (Murici) diferem entre as duas localidades, no mesmo município, sendo mais evidenciadas quanto ao tamanho dos frutos e das sementes. Inúmeros fatores podem influenciar nas diferenças entre essas características físicas como tipo de solo de cada local, precipitação pluvial, temperatura, variações topográficas e condições edafoclimáticas gerais. Cada variável influencia de uma forma na densidade, frequência e dominância tanto da espécie em si como de seus frutos. Outro fator importante que faz diferir no desenvolvimento dos frutos é a forma de manejo que o produtor impõe sobre a planta. Pois, em uma área na qual há a poda das matrizes, onde é feito o raleamento da mesma, a espécie vegetal tende a se desenvolver mais e, consequentemente, produzir mais e maiores frutos. Logo, indivíduos geneticamente idênticos podem se desenvolver de forma desigual em ambientes distintos.

\begin{tabular}{llllll}
\hline Caminhos de Geografia & Uberlândia - MG & v. 19, n. 68 & Dez/2018 & p. 36-45 & Página 37
\end{tabular}


Caracterização física dos frutos e sementes da espécie byrsonima gardneriana a. juss no semiárido alagoano
Élida Monique da Costa Santos; Kallianna Dantas Araujo; Mayara Andrade Souza; Ana Beatriz da Silva; Elba dos Santos Lira; Danúbia Lins Gomes; João Gomes da Costa

Cabe mencionar que o Murici é encontrado em larga distribuição na região de Olho D'Água do Casado, apresentando elevado potencial como fonte de alimentação humana, havendo em sua composição, dentre outros compostos, agentes antioxidantes e vitamina C. Através deste potencial existente, já verificado por Santos (2016), a espécie supracitada pode servir como fonte de renda economicamente viável para a população. No entanto, o conhecimento de seu potencial pelos moradores locais, ainda é reduzido. O quê, aliado a escassez de pesquisas sobre as potencialidades de espécies nativas como o Murici, oculta seu grande potencial de utilização (SOUZA et al., 2012) para fins medicinais e alimentícios. Contudo, esta pesquisa serve de embasamento para melhor entender como se da à dinâmica do desenvolvimento dos frutos do Murici no ambiente, e assim, cultivá-lo e/ou mantê-lo de forma a obter melhores desempenhos dos frutos. Além de apresentar elevada importância no âmbito social, ambiental e acadêmico, levando em consideração que não foram encontrados trabalhos referentes à caracterização física desta espécie, sendo assim um trabalho de caráter pioneiro.

Diante disso, o presente estudo objetivou analisar as características físicas dos frutos e sementes da espécie Byrsonima gardneriana A. Juss (Murici) em duas localidades, em Olho D'Água do Casado, Alagoas. Grande parte da população não tem conhecimento das potencialidades que este pequeno fruto apresenta e, em paralelo a isto, não costumam desenvolver algum tipo de manejo sobre a espécie, cabendo mencionar que o Murici pode se desenvolver de diferentes maneiras dependendo de onde está localizado e de como é tratado. Neste trabalho foi constatado que os frutos do Sítio Pudrinhos são mais desenvolvidos quando comparados aos do Sítio Alto. Visando as potencialidades que o Murici oferece, faz-se necessário que os produtores locais sejam instruídos a respeito do tipo de manejo para que haja melhor desenvolvimento da espécie e, consequentemente, maior disponibilidade dos frutos, tudo isso em consonância com a manutenção da biodiversidade.

\section{METODOLOGIA}

\section{CARACTERIZAÇÃO DA ÁREA DE ESTUDO}

A pesquisa foi realizada em Olho D’Água do Casado, Mesorregião Geográfica do Sertão Alagoano e Microrregião Geográfica Alagoana do Sertão do São Francisco (Figura 1), nas coordenadas geográficas $10^{\circ} 03^{\prime} 30^{\prime S}$ e $36^{\circ} 49^{\prime} 00^{\prime \prime} \mathrm{O}$, na altitude de 230 m (ALAGOAS, 2015), ocupando uma área de $322,264 \mathrm{~km}^{2}$ (IBGE, 2018).

Figura 1: Localização do município de Olho D’Água do Casado, Alagoas.

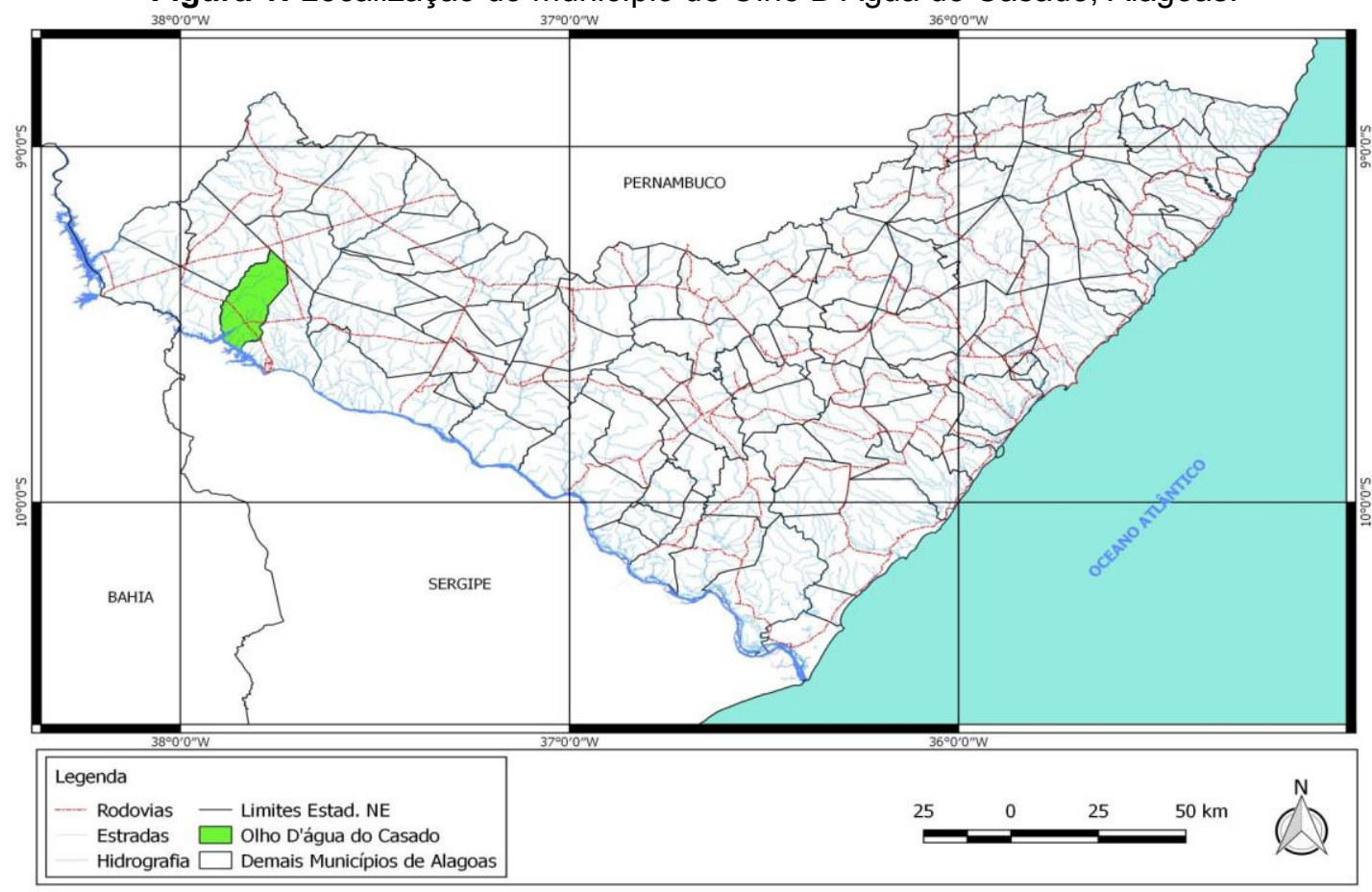

Fonte: SANTOS (2017). 
Caracterização física dos frutos e sementes da espécie byrsonima gardneriana a. juss no semiárido alagoano
Élida Monique da Costa Santos; Kallianna Dantas Araujo; Mayara Andrade Souza; Ana Beatriz da Silva; Elba dos Santos Lira; Danúbia Lins Gomes; João Gomes da Costa

O clima do local da pesquisa é BSh-Tropical Semiárido, segundo Köppen (LIMA, 1977) com período chuvoso de novembro a abril (MASCARENHAS et al., 2005), apresentando precipitação pluvial de $545,6 \mathrm{~mm} / \mathrm{ano}$, temperatura do ar média anual de $25,6{ }^{\circ} \mathrm{C}$ e umidade relativa de $74,4 \%$ (UFCG, 2018). O município de Olho D’Água do Casado está localizado na Depressão Sertaneja, que abrange uma superfície pediplanada do Baixo São Francisco, apresentando na parte Sul um relevo suavemente inclinado dando origem a uma cuesta cortada pelo Riacho do Talhado e seus afluentes, e na parte Norte estão presentes os relevos residuais (inselbergs). Apesar da predominância das rochas cristalinas, há também a presença de rochas sedimentares que formam os paredões ao longo do rio São Francisco (MASCARENHAS et al., 2005).

No município, nas áreas de patamares elevados e relevo suave ondulado, os solos predominantes são Planossolos. Já nos topos e vertentes predominam os Luvissolos e Argissolos, o primeiro apresentando-se raso e de fertilidade alta e o segundo de fertilidade natural média. Os Neossolos (rasos, pedregosos e de fertilidade natural média) ocorrem nos locais de maciços residuais (EMBRAPA, 2012). A vegetação predominante é a Caatinga Hipoxerófila com trechos de Floresta Caducifólia, caracterizadas por formações xerófilas, lenhosas, decíduas, em geral espinhosas (SANTANA e SOUTO, 2006), com ocorrência (além da Byrsonima gardneriana A. Juss (Murici)) de espécies como Aspidosperma pyrifolium Mart. (Pereiro), Pityrocarpa moniliformis (Benth) Luckow R. W. Jobson (Angelim), Tabubeia sp. (Pau d'arco) (SOUZA, 2011).

\section{LOCAL DA PESQUISA}

Foram selecionados dois pontos em área de Caatinga $(\mathrm{P} 1=$ Sítio Alto e $\mathrm{P} 2=$ Sítio Pudrinhos $)$, no município de Olho D'Água do Casado, para a coleta dos frutos para realização da pesquisa sobre as características físicas dos frutos e sementes da espécie $B$. gardneriana (Murici). Os pontos estudados, são caracterizados como áreas de caatinga nativa utilizada também como área de cultivo agrícola durante o período chuvoso na região. Em ambos locais estudados, há a conservação das matrizes nativas de Murici, estas sendo exploradas para fins alimentícios por parte da comunidade local no município de Olho D'Água do Casado. Além de nos dois locais, os proprietários terem criação de animais (aves).

As árvores nativas da espécie $B$. gardneriana que são encontradas nesses pontos apresentam matrizes de porte elevado, podendo chegar até $6 \mathrm{~m}$ de altura. As copas das matrizes são espaças e apresentam uma grande quantidade de frutos durante o período de frutificação, notadamente quando há eventos de precipitação contínua, proporcionando melhores condições para o desenvolvimento das fenofases desta espécie durante 0 ano.

Cabe destacar que no ponto 1 (Sítio Alto) as matrizes de Murici existentes não são submetidas a nenhum tipo de manejo. Já no ponto 2 (Sítio Pudrinhos), há maior cuidado com a área, pois o produtor pratica o raleamento da mesma e a poda da planta, condições que são propícias para o desenvolvimento do Murici.

\section{AQUISIÇÃO DA EXSICATA DA ESPÉCIE BYRSONIMA GARDNERIANA A. JUSS (MURICI)}

Para a aquisição da exsicata coletou-se parte do material vegetal da espécie como galho/ramo com folhas, botão floral, flor e frutos, os quais foram inseridos em prensa de madeira (Figuras 2A a 2C), sendo submetidos à secagem em estufa com circulação de ar a $65{ }^{\circ} \mathrm{C}$ por 72 horas, para identificação cuja exsicata corresponde ao no 15722. O material foi depositado no Herbário Jayme Coelho de Moraes (EAN), da Universidade Federal da Paraíba.

\section{ANÁLISES FÍSICAS DOS FRUTOS E SEMENTES}

Nas épocas de frutificação da espécie (julho e agosto/2014) foram coletadas amostras de frutos maduros para as avaliações físicas (Figuras 3A a 3C), sendo selecionadas 250 unidades destes e separados manualmente os sadios, inteiros e sem deformação. 
Caracterização física dos frutos e sementes da espécie byrsonima gardneriana a. juss no semiárido alagoano
Élida Monique da Costa Santos; Kallianna Dantas Araujo; Mayara Andrade Souza; Ana Beatriz da Silva; Elba dos Santos Lira; Danúbia Lins Gomes; João Gomes da Costa

Figura 2: Coleta dos galhos de Byrsonima gardneriana A. Juss (Murici) (A), exsicata da espécie (B) e prensa de madeira utilizada no campo $(C)$.
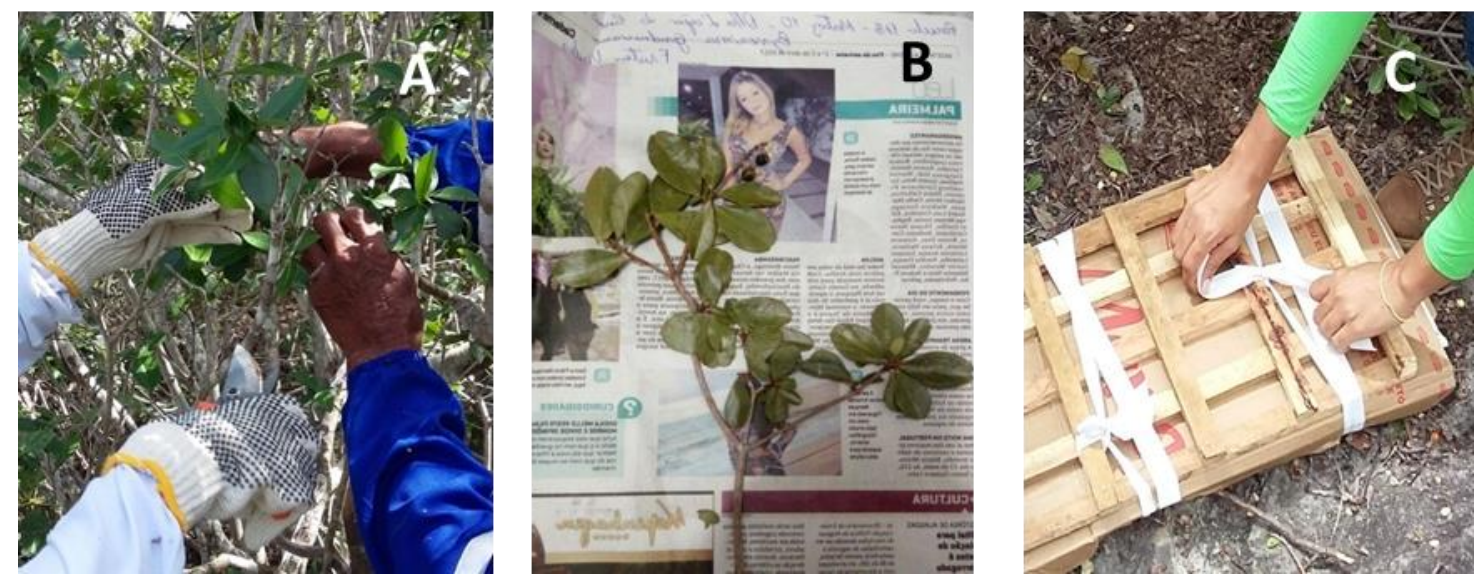

Fonte: Os autores (2014).

Figura 3: Matriz de Murici (Byrsonima gardneriana A. Juss) (A), coleta dos frutos (B) e frutos coletados e armazenados em sacos plásticos e caixa de isopor (C).
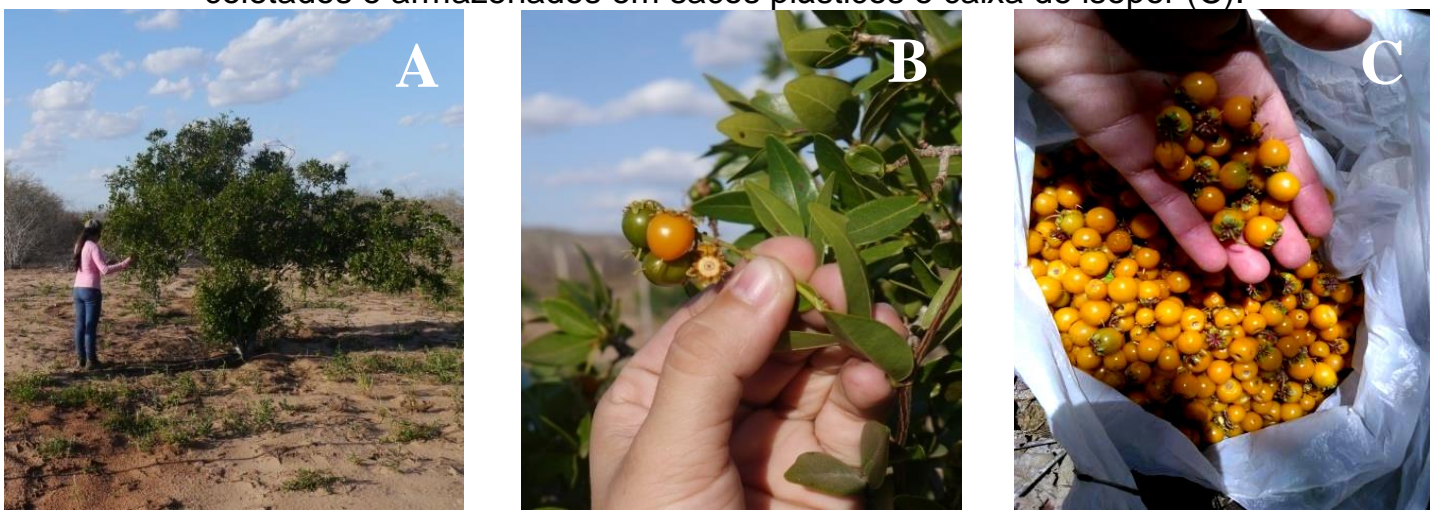

Fonte: Os autores (2014).

Dos frutos previamente selecionados, foi retirada uma amostra de 150 unidades, para mensuração do peso dos frutos (g) (Figura 4A) e peso das sementes (g) (Figura 4B), utilizando balança semianalítica de precisão com quatro casas decimais, rendimento de polpa e casca (\%), rendimento das sementes (\%), além de comprimento e diâmetro dos frutos e das sementes, medidos com paquímetro manual (Figura 4C). Para obtenção da polpa (Figura 4D) os frutos foram ralados em peneiras de aço, com malha de $1 \times 1 \mathrm{~mm}$. Devido ao pequeno tamanho do fruto, não houve separação da polpa e casca.

O rendimento de polpa e casca foi obtido pela equação:

$\mathrm{RPC}=\frac{\mathrm{Ppc}}{\mathrm{PFi}} \times 100$

em que:

$\mathrm{RPC}=$ Rendimento de polpa e casca;

$\mathrm{Ppc}=$ Peso de polpa e casca;

$\mathrm{Pfi}=$ Peso do fruto individual.

O rendimento das sementes foi calculado pela equação:

$\mathrm{RS}=\frac{\mathrm{P}_{5}}{\mathrm{PF}_{\mathrm{I}}} \times 100$ 
em que:

$\mathrm{RS}=$ Rendimento da semente;

Ps = Peso da semente;

$\mathrm{Pfi}=$ Peso do fruto individual.

Figura 4: Pesagem do fruto $(A)$, pesagem da semente $(B)$, medição do fruto $(C)$ e polpa obtida (polpa mais casca) (D) da espécie Byrsonima gardneriana A. Juss (Murici).
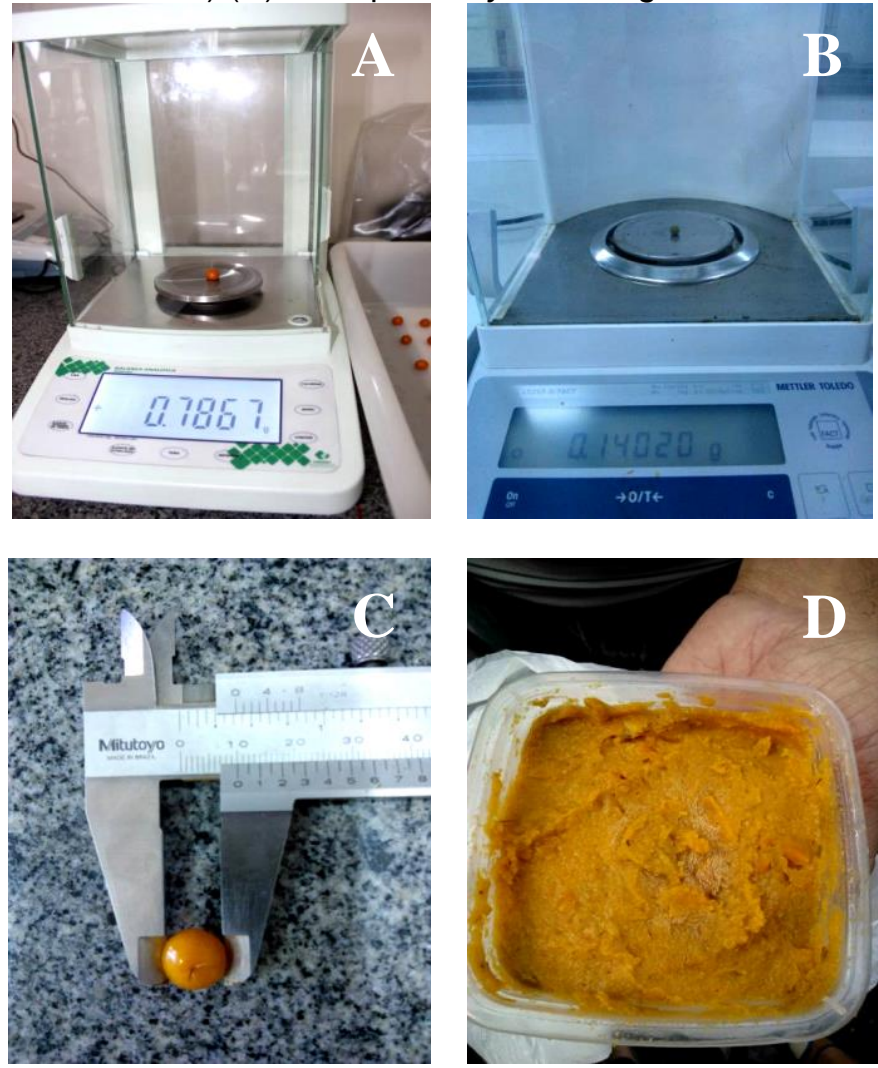

Fonte: Os autores (2014)

\section{ANÁLISE ESTATÍSTICA}

O delineamento foi inteiramente casualizado com dois tratamentos (população de B. gardneriana de cada local) e 150 repetições (frutos e sementes). Os dados obtidos na análise física foram submetidos à análise de variância e as médias foram comparadas pelo teste de Tukey a 5\% de probabilidade, utilizando o programa SAEG 9.1.

\section{RESULTADOS E DISCUSSÃO}

O fruto da espécie $B$. gardneriana, endêmica da Caatinga, apresenta pequena dimensão, é amareloalaranjado quando maduro, apresenta cheiro forte com polpa de sabor travoso, é oleoso e a semente é relativamente grande em relação ao tamanho do fruto.

Com exceção do rendimento de polpa e casca, houve diferença estatística $(P<0,01)$ para as demais variáveis. Analisando-se o diâmetro dos frutos da espécie $B$. gardneriana verificou-se que no P2 (Sítio Pudrinhos), os frutos apresentam diâmetro longitudinal e transversal superiores em relação ao P1 (Sítio Alto) (Tabela 1). 
Caracterização física dos frutos e sementes da espécie byrsonima gardneriana a. juss no semiárido alagoano
Élida Monique da Costa Santos; Kallianna Dantas Araujo; Mayara Andrade Souza; Ana Beatriz da Silva; Elba dos Santos Lira; Danúbia Lins Gomes; João Gomes da Costa

Tabela 1: Características físicas (média, desvio padrão e amplitude) de frutos e sementes da espécie Byrsonima gardneriana A. Juss (Murici) dos Pontos 1 (Sítio Alto) e 2 (Sítio Pudrinhos), em Olho D'Água do Casado, Alagoas

\begin{tabular}{lcccc}
\hline \multirow{2}{*}{ Variáveis/Locais } & \multicolumn{2}{c}{ P1 } & \multicolumn{2}{c}{ P2 } \\
\cline { 2 - 5 } & $\begin{array}{c}\text { Média e desvio } \\
\text { padrão }\end{array}$ & Amplitude & $\begin{array}{c}\text { Média e desvio } \\
\text { padrão }\end{array}$ & Amplitude \\
\hline DLF $(\mathrm{cm})$ & $0,78 \pm 0,0062 \mathrm{a}$ & $0,85-1,25$ & $1,03 \pm 0,0057 \mathrm{~b}$ & $0,60-1,00$ \\
DTF $(\mathrm{cm})$ & $0,69 \pm 0,0048 \mathrm{a}$ & $0,70-1,20$ & $0,82 \pm 0,0051 \mathrm{~b}$ & $0,50-0,80$ \\
DLS $(\mathrm{cm})$ & $0,55 \pm 0,0041 \mathrm{a}$ & $0,40-0,75$ & $0,70 \pm 0,0053 \mathrm{~b}$ & $0,60-0,90$ \\
DTS (cm) & $0,55 \pm 0,0041 \mathrm{a}$ & $0,45-0,85$ & $0,58 \pm 0,0039 \mathrm{~b}$ & $0,50-0,70$ \\
PFI $(\mathrm{g})$ & $0,56 \pm 0,0159 \mathrm{a}$ & $0,60-1,00$ & $0,83 \pm 0,0080 \mathrm{~b}$ & $0,63-0,19$ \\
PSI $(\mathrm{g})$ & $0,14 \pm 0,0018 \mathrm{a}$ & $0,08-0,90$ & $0,21 \pm 0,0023 \mathrm{~b}$ & $0,20-0,22$ \\
PPC (g) & $0,18 \pm 0,0062 \mathrm{a}$ & $0,07-0,94$ & $0,28 \pm 0,0073 \mathrm{~b}$ & $0,10-0,89$ \\
RPC (\%) & $32,99 \pm 1,2039 \mathrm{a}$ & $9,07-182$ & $32,69 \pm 0,7170 \mathrm{a}$ & $15,72-102,16$ \\
RS $(\%)$ & $26,74 \pm 0,4953 \mathrm{a}$ & $11,42-37,28$ & $24,61 \pm 0,1778 \mathrm{~b}$ & $34,62-18,93$
\end{tabular}

DLF: Diâmetro Longitudinal do Fruto; DTF: Diâmetro Transversal do Fruto; DLS: Diâmetro Longitudinal da Semente; DTS: Diâmetro Transversal da Semente; PFI: Peso do Fruto Individual; PSI: Peso da Semente Individual; PPC: Peso de Polpa e Casca; RPC: Rendimento Polpa e Casca; RS: Rendimento das Sementes. Médias seguidas da mesma letra na mesma linha não diferem estatisticamente ao nível de $1 \%$ de probabilidade pelo teste de Tukey.

Fonte: Os autores (2015).

No P1 o diâmetro longitudinal dos frutos apresentou variação entre 0,60 e $1,00 \mathrm{~cm}$ e de 0,85 a 1,25 $\mathrm{cm}$ no P2. Já o diâmetro transversal oscilou de 0,50 a $0,80 \mathrm{~cm}$ e de 0,70 a $1,20 \mathrm{~cm}$, nos locais $\mathrm{P} 1 \mathrm{e}$ P2, respectivamente (Tabela 1). Esses valores foram similares aos encontrados por Araújo et al. (2009), em pesquisa realizada em Maceió, Alagoas, com a espécie B. verbascifolia, cujos valores de diâmetros longitudinal e transversal dos frutos corresponderam a 0,85 e $0,74 \mathrm{~cm}$, respectivamente. $O$ conhecimento das características físicas de frutos do mesmo gênero e até mesmo da mesma espécie, apresentam importância no sentido de que dessa forma pode ser selecionada, para a indústria alimentícia, por exemplo, a espécie (ou a localidade) que apresenta frutos de maiores dimensões, já que assim, o rendimento do mesmo vai ser maior. Ao observar os dados descritos e, embora ambas as espécies sejam visualmente similares quanto à forma da folha, flor e fruto, a espécie $B$. gardneriana apresenta um potencial maior ao que foi citado anteriormente.

Os valores para comprimento (diâmetro longitudinal) e diâmetro (diâmetro transversal) dos frutos também foram semelhantes aos encontrados por Gusmão et al. (2006) que, estudando $B$. verbascifolia em uma área de Cerrado no Norte de Minas Gerais, constataram para os frutos a média de $1,00 \mathrm{~cm}$ de comprimento e $1,19 \mathrm{~cm}$ de diâmetro. Os autores afirmam ainda que essas diferenças encontradas entre os tamanhos dos frutos devem-se às influências microclimáticas e edáficas existentes ao longo da área onde estes foram coletados, como o conteúdo de água e minerais disponibilizados para cada matriz de Murici.

Pode-se observar a diferença de tamanho dos frutos entre os dois locais estudados. Ressaltando-se que nos dois pontos, as matrizes selecionadas para a coleta dos frutos são nativas, e em nenhuma das áreas há presença de irrigação, o quê aumentaria o percentual de água no solo interferindo no desenvolvimento da árvore e consequentemente, dos frutos, pois foi constatado por Lira (2016) que esta espécie responde rapidamente à quantidade de água que Ihe é disponibilizada. Outra questão que merece destaque é que no ponto 1 as matrizes de Murici não recebem nenhum tipo de manejo, já no ponto 2 estas são submetidas a processos de poda e limpeza (raleamento) da área onde estão inseridas. Práticas como essas fazem com que as matrizes do segundo local se desenvolvam mais e produzam maior número de frutos. Além disso, ambas as áreas apresentam presença de material foliar no solo, proporcionando melhores condições deste, devido à decomposição do material através dos organismos edáficos. De acordo com Borchartt (2013) em ecossistemas naturais preservados a quantidade de material foliar depositado no solo e sua decomposição, contribuem para a elevação dos teores de matéria orgânica (esta se apresentando como importante indicador da qualidade do solo), influenciando nas propriedades químicas, físicas e biológicas do ambiente edáfico. Cabendo mencionar que todos esses fatores são importantes para o desenvolvimento das fenofases de quaisquer espécies vegetais. 
Caracterização física dos frutos e sementes da espécie byrsonima gardneriana a. juss no semiárido alagoano
Élida Monique da Costa Santos; Kallianna Dantas Araujo; Mayara Andrade Souza; Ana Beatriz da Silva; Elba dos Santos Lira; Danúbia Lins Gomes; João Gomes da Costa

Em relação ao peso dos frutos constatou-se variação entre $0,33 \mathrm{~g}$ no $\mathrm{P} 1$ e $1,19 \mathrm{~g}$ no P2 (Tabela 1). É importante destacar que os frutos de uma mesma espécie podem apresentar variações de tamanho, peso e rendimento, em decorrência da variação de temperatura, conteúdo de água do solo, precipitação, minerais presentes no solo, que interferem no meio em que a espécie se encontra, agindo na sua composição. Santos et al. (2009) afirmam que as variações no peso dos frutos podem decorrer da variabilidade genética ou de variações ambientais decorrentes das diferentes localidades geográficas, nas quais os frutos estão localizados.

Analisando-se o peso da polpa e casca registrou-se oscilação entre $0,06 \mathrm{~g}$ (P1) e 0,89 g (P2), resultando em rendimento da polpa e casca com valores aproximados em relação aos locais (Tabela 1), não havendo diferença significativa, em virtude dos diâmetros longitudinais e transversais das sementes que apresentaram valores próximos aos dos frutos, culminando no baixo rendimento da polpa (Tabela 1).

Morzelle et al. (2015), no Estado do Mato Grosso, analisando a espécie B. crassifolia verificaram que o rendimento da polpa comestível do fruto, que compreende a casca e polpa, correspondeu a 75,99\%, sendo superior aos dados obtidos nesta pesquisa com B. gardneriana. Essa discrepância nos valores pode ser atribuída, devido aos mesmos serem de espécies distintas. De acordo com Nascimento et al. (2014), o peso de polpa e casca é o atributo físico de maior importância para a exploração econômica industrial, notadamente no que se refere ao processamento de frutos. É importante ressaltar que a avaliação do rendimento de polpa e casca foi feita sem adição de água, cujo material foi retirado do fruto e logo em seguida mensurado o peso individual da polpa de cada fruto, já que quando há adição de água à polpa, há um rendimento maior do produto.

Ao avaliar o rendimento das sementes os valores verificados para os dois locais foram aproximados, totalizando $24,61 \%$ no P2 e $26,74 \%$ no P1. Embora os valores referentes ao rendimento das sementes tenham sido elevados, o fruto apresenta potencial para a indústria alimentícia mesmo não apresentando elevado rendimento da polpa (Figura 5), pois é um fruto que apresenta compostos muito importantes para a alimentação e utilização humana, com potencial antioxidante e presença de compostos fenólicos (SANTOS, 2016), além da presença de vitamina C.

As sementes no P2 apresentaram diâmetros longitudinal e transversal médio de 0,70 e 0,58 cm, respectivamente, sendo superiores aos verificados no $\mathrm{P} 1$, cujo resultado para ambas as variáveis correspondeu à média de $0,55 \mathrm{~cm}$. Esses resultados foram inferiores aos encontrados por Gusmão et al. (2006), que estudando os endocarpos (sementes) de B. verbascifolia, constataram a média de 0,66 e 0,88 cm de comprimento (diâmetro longitudinal) e diâmentro (diâmetro transversal), respectivamente. A variação do diâmetro longitudinal das sementes correspondeu a 0,40 e $0,75 \mathrm{~cm}$ no P2 e de 0,60 a $0,90 \mathrm{~cm}$ no P1. O diâmetro transversal oscilou entre 0,45 e $0,85 \mathrm{~cm}$ no $\mathrm{P} 1$ e de 0,50 a $0,70 \mathrm{~cm}$ no P2 (Tabela 1). Registrou-se variação para o peso das sementes da espécie $B$. gardneriana entre os locais, com 0,08 g (P1) e 0,31 g (P2), apresentando como peso médio 0,21 g no P2 e $0,14 \mathrm{~g}$ no P1 (Tabela 1 ).

Cabe destacar que em relação às sementes de Malpighiaceae, família à qual pertence o gênero Byrsonima, Rolim (2009) enfatiza que poucas espécies foram estudadas em detalhe, principalmente tratando-se deste gênero, havendo também escassez de trabalhos referentes à parte física das sementes da espécie B. gardneriana destacando-se a importância deste trabalho.

É importante ressaltar que o fruto da espécie $B$. gardneriana ainda é pouco utilizado pela população local, embora seja considerado pelos produtores rurais como fonte de forragem para os ruminantes, sendo também consumido e comercializado nas formas in natura e em produto como flau, também conhecido como dindin, sacolé, dida, dudú, gelinho, dentre outros, devido ao pouco conhecimento das potencialidades do mesmo. No entanto, o fruto tem potencialidade para o consumo humano e pode ser comercializado de outras formas, em produtos como trufa, mousse, licor, brigadeiro, doce e geleia (SANTOS, 2016), sendo este último, a melhor forma de conservar o produto por mais tempo.

\section{CONSIDERAÇÕES FINAIS}

Os frutos e sementes da espécie Byrsonima gardneriana A. Juss do Ponto 2 (Sítio Pudrinhos) são mais desenvolvidos em relação ao Ponto 1 (Sítio Alto) para as variáveis Diâmetro Longitudinal e Transversal dos frutos e sementes, Peso dos frutos e das sementes e Peso de polpa e casca, atribuída a variabilidade edafoclimática existentes entre os locais estudados e ao tipo de manejo que cada produtor exerce sobre a espécie nessas áreas;

\begin{tabular}{llllll}
\hline Caminhos de Geografia & Uberlândia - MG & v. 19, n. 68 & Dez/2018 & p. 36-45 & Página 43
\end{tabular}


Caracterização física dos frutos e sementes da espécie byrsonima gardneriana a. juss no semiárido alagoano
Élida Monique da Costa Santos; Kallianna Dantas Araujo; Mayara Andrade Souza; Ana Beatriz da Silva; Elba dos Santos Lira; Danúbia Lins Gomes; João Gomes da Costa

A espécie $B$. gardneriana possui semente grande em relação ao tamanho do fruto, e não há alto rendimento de polpa. Entretanto, esta circunstância não diminui sua importância para a indústria alimentícia, pois, essa característica pode ser melhorada por intermédio da seleção de plantas com frutos que apresentam maior rendimento de polpa;

Grande parte da população não tem conhecimento das potencialidades que este pequeno fruto apresenta, e que quando utilizado de maneira correta pode ser uma alternativa de fonte de renda para a população;

Visando essas potencialidades que o Murici oferece, faz-se necessário que os produtores locais sejam instruídos a respeito do tipo de manejo para que haja um melhor desenvolvimento da espécie e, consequentemente, maior disponibilidade dos frutos, tudo isso em consonância com a manutenção da biodiversidade.

\section{AGRADECIMENTOS}

À CAPES, pela concessão da bolsa para a realização desta pesquisa; À UFAL e ao IGDEMA pela oportunidade de desenvolvimento da pesquisa no Programa de Pós-Graduação em Geografia; Ao Grupo de Pesquisa Biogeografia e Sustentabilidade Ambiental e ao Laboratório de Ecogeografia e Sustentabilidade Ambiental - Labesa da UFAL, pelo auxílio nas atividades de campo e laboratório; Ao Laboratório de Biotecnologia - Embrapa Tabuleiros Costeiros, pelo auxílio durante a pesquisa e realização das análises físicas; Ao Laboratório de Pesquisas em Recursos Naturais - LPqRN da UFAL, pela disponibilidade do local para desenvolvimento de parte dos testes; Aos senhores Agaiton Gonçalves de Souza e Adalberto Inácio de França com toda ajuda disponibilizada através de seus conhecimentos e experiências com a Caatinga.

\section{REFERÊNCIAS}

ALAGOAS. Secretaria de Estado do Planejamento e Desenvolvimento Econômico. Perfil Municipal: Olho D’Água do Casado. Maceió: 2015.

ARAÚJO, R. R. de. et al. Caracterização biométrica de frutos e sementes de genótipos de Murici (Byrsonimaverbascifolia(L.) Rich.) do Tabuleiro Costeiro de Alagoas. Revista Caatinga, v. 22, p. 224228, 2009.

BENINI, E. B. et al. Valorização da flora nativa quanto ao potencial fitoterápico. Revista Destaques Acadêmicos, v. 2, p. 11-18, 2010.

BORCHARTT, L. Atributos físicos e químicos de uma Topossequência e ciclagem de nutrients em areas de mata native e reflorestada.Tese (Doutorado em Agronomia) - Areia: UFPB. 2013.

CHITARRA, M. I. F.; CHITARRA, A. B. Pós-colheita de frutos e hortaliças: fisiologia e manuseio. 1. ed. Lavras: ESAL/FAEPE, 1990.

DRUMOND, M. A. et al. Avaliação e identificações de ações prioritárias para a conservação, utilização sustentável e repartição dos benefícios da biodiversidade do bioma Caatinga: Estratégias para o Uso Sustentável da Biodiversidade da Caatinga. 1. ed. Petrolina: Embrapa Semiárido, 2000. (Documento Técnico).

EMPRESA BRASILEIRA DE PESQUISAS AGROPECUÁRIAS - EMBRAPA. Zoneamento agroecológico do Estado de Alagoas: Levantamento de reconhecimento de baixa e média intensidade dos solos do Estado de Alagoas. 1. ed. Rio de Janeiro: Embrapa Solos, 2012.

GUSMÃO, E.; VIEIRA, F. A.; FONSECA, E. M. Biometria de frutos e endocarpo de murici (ByrsonimaverbascifoliaRich. ex. A. Juss). Cerne, v. 12, p. 84-91, 2006.

IBGE - Instituto Brasileiro de Geografia e Estatística.IBGE CIDADES. 2018. Disponível em: <http://www.cidades.ibge.gov.br/>. Acesso em: 16 de julho de 2018.

LIMA, I. F. Fundamentos geográficos do meio físico do Estado de Alagoas. 1. ed. Maceió: Governo do Estado de Alagoas/SEPLAN/SUDENE, 1977. (Série: Estudo de Regionalização).

LIRA, E. dos S. Análise espaço-temporal da estrutura e fenologia da espécie Byrsonima gardneriana A. Juss em area de Caatinga no Semiárido Alagoano. Dissertação (Mestrado em Geografia) - Maceió: UFAL. 2016.

$\begin{array}{llllll}\text { Caminhos de Geografia } & \text { Uberlândia - MG } & \text { v. 19, n. } 68 & \text { Dez/2018 } & \text { p. 36-45 } & \text { Página } 44\end{array}$


Caracterização física dos frutos e sementes da espécie byrsonima gardneriana a. juss no semiárido alagoano
Élida Monique da Costa Santos; Kallianna Dantas Araujo; Mayara Andrade Souza; Ana Beatriz da Silva; Elba dos Santos Lira; Danúbia Lins Gomes; João Gomes da Costa

MASCARENHAS, J. de C.; BELTRÃO, B. A., SOUZA JUNIOR, L. C. de. Projeto cadastro de fontes de abastecimento por água subterrânea: diagnóstico do município de Olho D' Água do Casado, Estado de Alagoas. 1. ed. Recife: CPRM/PRODEEM, 2005.

MORZELLE, M. C. et al. Caracterização química e física de frutos de curriola, gabiroba e murici provenientes do Cerrado brasileiro. Revista Brasileira de Fruticultura, v. 37, p. 96-103, 2015. https://doi.org/10.1590/0100-2945-036/14

NASCIMENTO, R. S. M.; CARDOSO, J. A.; COCOZZA, F. D. M. Caracterização física e físicoquímica de frutos de mangabeira (Hancorniaspeciosa Gomes) no oeste da Bahia. Revista Brasileira de Engenharia Agrícola e Ambiental, v.18, p.856-860, 2014. https://doi.org/10.1590/18071929/agriambi.v18n08p856-860

ROLIM, T. L. Estudo fitoquímico de Byrsonimagardneriana A. Juss (Malpighiaceae).Dissertação (Mestrado em Produtos Naturais e Sintéticos Bioativos) - João Pessoa: UFPB. 2009.

SANTANA, J. A. da S.; SOUTO, J. S. Diversidade e estrutura fitossociológica da caatinga na Estação Ecológica do Seridó-RN. Revista de Biologia e Ciências da Terra, v. 6, p. 232-242, 2006.

SANTOS, É. M. da C. Importância socioeconômica da espécie Byrsonimagardneriana A. Juss (Murici) como alternativa de renda complementar para a população do semiárido Alagoano. Dissertação (Mestrado em Geografia) - Maceió: UFAL. 2016.

SANTOS, F. S. et al. Biometria e qualidade fisiológica de sementes de diferentes matrizes de Tabebuia chrysotricha (Mart. ex A. DC.) Standl. ScientiaForestalis, v.37, p.163-173, 2009.

SEIXAS, E. N. C. et al. Biologia reprodutiva e propriedades químico farmacológicas de ByrsonimaRich. ExKunth (Malpighiaceae) no Nordeste - Brasil. Caderno de Cultura e Ciência, v. 10, p. 7-16, 2011. https://doi.org/10.14295/cad.cult.cienc.v10i2.399

SOUZA, M. A. et al. Análise sensorial de alimentos enriquecidos com polpa de Murici (ByrsonimagardnerianaA. Juss). In: XXII CONGRESSO BRASILEIRO DE FRUTICULTURA, 22, 2012, Bento Gonçalves. Anais... Bento Gonçalves: Sociedade Brasileira de Fruticultura, 2012. p. 49344937.

SOUZA, M. A. Fitossociologia em áreas de caatinga e conhecimento etnobotânico do murici (ByrsonimagardnerianaA. Juss.), Semiárido Alagoano. Dissertação (Mestrado em Agronomia) Areia: UFPB. 2011.

UFCG - UNIVERSIDADE FEDERAL DE CAMPINA GRANDE. Dados climatológicos do Estado de Alagoas: Campina Grande: UFCG-CTRN, 2018. Disponível em: <http//www.dca.ufcg.edu.br> Acesso em: 16 de maio de 2018.

Recebido em: 12/10/2017

Aceito para publicação em: 11/09/2018 